\title{
Interplay of Non-Relativistic and Relativistic Effects in Neutrino Oscillations
}

\author{
D. V. Ahluwalia * and T. Goldman ${ }^{\dagger}$ \\ Los Alamos National Laboratory, Los Alamos, NM 87545, USA
}

\begin{abstract}
A theoretical structure that involves neutrino mass eigenstates at non relativistic as well as relativistic energies is presented. Using this framework, we find that if the particle $\mathrm{X}$, with mass $33.9 \mathrm{MeV}$, of the KARMEN collaboration anomaly is identified with the third neutrino mass eigenstate, then the present limit of $23 \mathrm{MeV}$ upper bound on the tau neutrino mass implies $\left|U_{\tau 3}\right|<0.82$.
\end{abstract}

*E-mail: av@p25hp.lanl.gov.

${ }^{\dagger} \mathrm{E}-$ mail: goldman@t5.lanl.gov. 


\section{INTRODUCTION}

The existing indications of neutrino oscillations [1-3] arise from data for neutrino energies in the sub-MeV to GeV range. The neutrino oscillation phenomenology within the standard three-flavor framework is developed on the basic assumption that the neutrino mass eigenstates that are superposed to yield the neutrino with flavor eigenstates are relativistic. This assumption is supported by the cosmological argument [4] that the sum of the neutrino masses have an upper bound of about $30 \mathrm{eV}$

$$
\sum_{\ell} m\left(\nu_{\ell}\right) \leq 30 \mathrm{eV}
$$

However, very recent astronomical observations [577] raise potentially serious questions on the validity of the standard cosmological model. First [5, 6], the UC Berkeley's Extreme Ultraviolet Explorer satellite's observations of the Coma cluster of galaxies indicates that this cluster of galaxies may contain a submegakelvin cloud of $\sim 10^{13} M_{\odot}$ of baryonic gas. Second [7], the discovery by the German X-ray satellite Rosat in which a sample of 24 Seyfert galaxies contained 12 that were accompanied by a pairs of $\mathrm{X}$-ray sources, almost certain to be high red shift quasars, aligned on either side of the galaxy. These observations and the associated interpretations, if correct, may place severe questions to cosmological models that depend on the ratio of photon to baryonic density in the universe and its size.

We, therefore, consider it reasonable to relax the cosmological constraint (1) completely and explore the resulting consequences for three-flavor neutrino oscillation framework. The latest kinematic limits on neutrino masses are much less severe [8,9]:

$$
\begin{aligned}
& m\left(\nu_{\tau}\right)<23 \mathrm{MeV} \\
& m\left(\nu_{\mu}\right)<0.17 \mathrm{MeV} \\
& m\left(\nu_{e}\right)<10-15 \mathrm{eV} .
\end{aligned}
$$

The existing and the proposed neutrino oscillation experiments involve neutrino energies from a fraction of a $\mathrm{MeV}$ to several hundred $\mathrm{GeV}$ for the upper-energy end of the neutrino beams. It remains possible that for some of the experiments (or a sector of an experiment), the mass eigenstates $\left|\nu_{2}\right\rangle$ and/or $\left|\nu_{3}\right\rangle$ are non relativistic. Towards the end of understanding such a possible situation we now consider the interplay of non-relativistic and relativistic mass eigenstates in three-flavor neutrino oscillation framework.

To establish the notational and conceptual context, we begin with a brief review of the the standard three-flavor neutrino oscillation framework.

\section{FRAMEWORK A: THE STANDARD THREE-FLAVOR NEUTRINO OSCILLATION FRAMEWORK}

The standard three-flavor neutrino oscillation framework begins with the assumptions that $[10$ 12]

1. There is no $\mathrm{CP}$ violation in the neutrino sector.

2. Both the flavor and mass eigenstates are relativistic in the laboratory frame. 
3. All neutrino mass eigenstates are stable.

4. The weak flavor eigenstates $\left|\nu_{e}\right\rangle,\left|\nu_{\mu}\right\rangle,\left|\nu_{\tau}\right\rangle$ are not identical to the mass eigenstates:

$$
\left|\nu_{\ell}\right\rangle=\sum_{\jmath} U_{\ell_{\jmath}}\left|\nu_{\jmath}\right\rangle
$$

In the Maiani representation [13], with $\mathrm{CP}$ phase $\delta$ set equal to zero, the unitary neutrino-mixing matrix reads:

$$
U(\theta, \beta, \psi)=\left(\begin{array}{ccc}
c_{\theta} c_{\beta} & s_{\theta} c_{\beta} & s_{\beta} \\
-c_{\theta} s_{\beta} s_{\psi}-s_{\theta} c_{\psi} & c_{\theta} c_{\psi}-s_{\theta} s_{\beta} s_{\psi} & c_{\beta} s_{\psi} \\
-c_{\theta} s_{\beta} c_{\psi}+s_{\theta} s_{\psi} & -s_{\theta} s_{\beta} c_{\psi}-c_{\theta} s_{\psi} & c_{\beta} c_{\psi}
\end{array}\right)
$$

where $c_{\theta}=\cos (\theta), s_{\theta}=\sin (\theta)$, etc. The rows and columns are labeled as $U_{\ell \jmath}(\theta, \beta, \psi)$ with $\ell$ representing the neutrino-flavor eigenstates, $\ell=e, \mu, \tau$, and $\jmath=1,2,3$ denoting the three mass eigenstates with $m_{1}<m_{2}<m_{3}$.

Within this framework, if a neutrino flavor state $\left|\nu_{\ell}\right\rangle$, characterized by energy $E$, is created at $x=0$ and $t=0$ then the probability that one observes a neutrino state $\left|\nu_{\ell^{\prime}}\right\rangle$ at a neutrino-flavor sensitive detector at $x=L \simeq t$ is 14

$$
\begin{aligned}
& P_{\ell \ell^{\prime}}\left(E, L,\left\{\eta_{k}\right\}\right)=\delta_{\ell \ell^{\prime}}-4 U_{\ell^{\prime} 1} U_{\ell 1} U_{\ell^{\prime} 2} U_{\ell 2} \sin ^{2}\left(\varphi_{21}^{0}\right) \\
& \quad-4 U_{\ell^{\prime} 1} U_{\ell 1} U_{\ell^{\prime} 3} U_{\ell 3} \sin ^{2}\left(\varphi_{31}^{0}\right)-4 U_{\ell^{\prime} 2} U_{\ell 2} U_{\ell^{\prime} 3} U_{\ell 3} \sin ^{2}\left(\varphi_{32}^{0}\right),
\end{aligned}
$$

where the kinematic phase is defined as

$$
\varphi_{\jmath \imath}^{0}=2 \pi \frac{L}{\lambda_{\jmath \imath}^{\mathrm{osc}}} .
$$

The flavor-oscillation length is defined as

$$
\lambda_{\jmath}^{\mathrm{osc}}=\frac{2 \pi}{1.27} \frac{E}{\Delta m_{\jmath \imath}^{2}} .
$$

The five neutrino oscillation parameters $\left\{\eta_{k}\right\}$ that appear in Eq. (7) are the two mass squared differences and the three mixing angles: $\eta_{1}=\Delta m_{21}^{2}, \eta_{2}=\Delta m_{32}^{2}, \eta_{3}=\theta, \eta_{4}=\beta$, and $\eta_{5}=\psi$. The third mass squared difference is then given by $\Delta m_{31}^{2}=\Delta m_{21}^{2}+\Delta m_{32}^{2}$. Here, 1.27 is the usual factor that arises from expressing the neutrino energy $E$ in $\mathrm{MeV}$ (or GeV), the source-detector distance $L$ in meters (or kilometers), and the mass squared differences $\Delta m_{j k}^{2}$ in $\mathrm{eV}^{2}$.

The kinematic phase may also be written as: $\varphi_{\jmath \imath}^{0}=1.27 \Delta m_{\jmath \imath}^{2} \times(L / E)$. These kinematic phases may be modified for dynamical reasons [15]17.

For all three pairs of relativistic mass eigenstates, the relevant oscillation lengths are given by Eq. (9). Again, because $\Delta m_{31}^{2}=\Delta m_{21}^{2}+\Delta m_{32}^{2}$, there are only two independent length scales: $\lambda_{21}^{\text {osc }}$ and $\lambda_{32}^{\text {osc }}$. The third oscillation length is:

$$
\lambda_{31}^{\mathrm{osc}}=\frac{\lambda_{21}^{\mathrm{osc}} \lambda_{32}^{\mathrm{osc}}}{\lambda_{21}^{\mathrm{osc}}+\lambda_{32}^{\mathrm{osc}}}
$$


The physical origin of this lies in the assumption that all three mass eigenstates are relativistic and that neutrino oscillations are inherently a quantum mechanical interference phenomena.

Within the framework of the standard three-flavor neutrino oscillation framework one cannot simultaneously accommodate the known indications of the neutrino oscillations without questioning a set of experiments or the associated models (such as the standard solar model). One falls short of one length scale. If one assumes that all experiments, and the relevant theoretical understanding of the neutrino fluxes, are correct one is forced to invoke additional physics, most commonly taken to be the existence of a sterile neutrino [18].

In principle, there are six length scales in the three-flavor neutrino oscillation phenomenology. Three are associated with the three mass eigenstates. The other three, associated with life times of the mass eigenstates, are rendered irrelevant by the assumption of the stability of all three mass eigenstates. In addition, matter effects and gravitational radii in astrophysical environments contain additional length scales (see below on how gravitational radii may come into the picture).

A theoretical structure that contains in it (a) the standard three-flavor neutrino oscillation framework for the zenith angle dependent atmospheric neutrino anomaly (Energies in the GeV range), and (b) a three-flavor neutrino oscillation framework with one or two nonrelativistic mass eigenstates for part of the $\mathrm{MeV}$ data, provides an alternative hypothesis to the assumption of sterile neutrino.

For the sake of simplicity we shall maintain the assumption that all three neutrino mass eigenstates are stable.

\section{FRAMEWORK B: NEUTRINO OSCILLATIONS WITH A SUPERPOSITION OF TWO RELATIVISTIC AND ONE NON-RELATIVISTIC MASS EIGENSTATE}

Consider a physical situation where we have two relativistic, $\left|\nu_{1}\right\rangle$ and $\left|\nu_{2}\right\rangle$, neutrino mass eigenstates and one that is non-relativistic, $\left|\nu_{3}\right\rangle$.

At $t=0, x=0$, assume that a source creates a neutrino of flavor $\ell$

$$
\left|\nu_{\ell}\right\rangle=U_{\ell 1}\left|\nu_{1}\right\rangle+U_{\ell 2}\left|\nu_{2}\right\rangle+U_{\ell 3}\left|\nu_{3}\right\rangle
$$

The spatial envelope, which is assumed to be "sufficiently" narrow, associated with $\left\{\left|\nu_{1}\right\rangle,\left|\nu_{2}\right\rangle\right\}$ evolves towards the detector as $x \simeq t$, while the spatial envelope of $\left|\nu_{3}\right\rangle$ evolves towards the detector as

$$
x \simeq\left(p / m_{3}\right) t=\left(\frac{\sqrt{2 m_{3}\left(E-m_{3}\right)}}{m_{3}}\right) t .
$$

Therefore, the $\left\{\left|\nu_{1}\right\rangle,\left|\nu_{2}\right\rangle\right\}$ arrives at the detector at time $t_{I} \simeq L$, while the $\left|\nu_{3}\right\rangle$ arrives at

the detector at a later time $t_{I I} \simeq m_{3} L / \sqrt{2 m_{3}\left(E-m_{3}\right)}$. For the above considered energies and for a source-detector distance of a few tens (or greater) of meters the detected $\left|\nu_{\ell^{\prime}}\right\rangle$ has no overlap with $\left|\nu_{3}\right\rangle$ at the registered event at $t_{I}$, and similarly the detected $\left|\nu_{\ell^{\prime}}\right\rangle$ has no overlap with $\left\{\left|\nu_{1}\right\rangle,\left|\nu_{2}\right\rangle\right\}$ at the registered event at $t_{I I}$. With these observations at hand one can easily evaluate the modification to the neutrino oscillation probability (7). The modified expression reads: 


$$
\begin{aligned}
P_{\ell \ell^{\prime}}\left(E, L,\left\{\xi_{k}\right\}\right)=\left(U_{\ell^{\prime} 3} U_{\ell 3}\right)^{2}+ & {\left[\left(U_{\ell^{\prime} 1} U_{\ell 1}+U_{\ell^{\prime} 2} U_{\ell 2}\right)^{2}\right.} \\
& \left.-4 U_{\ell^{\prime} 1} U_{\ell 1} U_{\ell^{\prime} 2} U_{\ell 2} \sin ^{2}\left(\varphi_{21}^{0}\right)\right] .
\end{aligned}
$$

The first term on the rhs of the above equation is the contribution from the non-relativistic mass eigenstate to the $P_{\ell \ell^{\prime}}\left(E, L,\left\{\xi_{k}\right\}\right)$ at the $\nu_{\ell^{\prime}}$ event at time $t_{I I}$ while the second term is the contribution from the relativistic mass eigenstates to the $\nu_{\ell^{\prime}}$ event at the earlier time $t_{I}$. Contained in the above expression is the the fundamental "collapse of the wave packet" postulate of the orthodox interpretation of the quantum mechanics. That is, given a single $\nu_{\ell}$ emitted at the source, if the event occurs at $t_{I}$ no event occurs at $t_{I I}$, and vice versa.

There are several observations that one may make about the result (13). These observations follow.

I. The neutrino oscillation probability now contains only one length scale, $\xi_{1}=\Delta m_{21}^{2}$, $\xi_{2}=\theta, \xi_{3}=\beta$, and $\xi_{4}=\psi$. However, this loss of a length scale is related to a manifestly different expression for neutrino oscillation probabilities.

II. If $m_{3}$ is in the range of a fraction of an $\mathrm{MeV}$ to a few tens of $\mathrm{MeV}$, one cannot base the analysis of existing neutrino oscillation data in terms of Eq. (17), or Eq. (13), alone. For the zenith-angle dependence of the atmospheric neutrino anomaly data [3] neutrino energies in the $\mathrm{GeV}$ range are involved. This meets the requirements under which Eq. ([) is applicable. On the other hand, the physical situation for part of the LSND events (energy range between $20 \mathrm{MeV}$ and Michel spectrum cutoff of 52.8 Mev [2]), and the reactor experiments (average $\bar{\nu}_{e}$ energy about $5 \mathrm{MeV}$ [19 22]), the physical conditions for Eq. (13) may be satisfied.

III. For the solar-neutrino deficit it is conceivable that $m_{3}$ may be such that an energydependent transition takes place from the non-relativistic regime to relativistic regime, thus accounting for the apparent energy dependence of the solar-neutrino deficit in an unconventional manner. Consider the situation where the length scales are such that all $\sin ^{2}\left(\varphi_{\jmath \imath}^{0}\right)$ average to $1 / 2$. Then

$$
\begin{gathered}
P_{e e}[\text { Eq. }(7)]=1-2 U_{e 1}^{2} U_{e 2}^{2}-2 U_{e 1}^{2} U_{e 3}^{2}-2 U_{e 2}^{2} U_{e 3}^{2} \\
P_{e e}[\text { Eq. }(13)]=U_{e 1}^{4}+U_{e 2}^{4}+U_{e 3}^{4} .
\end{gathered}
$$

Exploiting unitarity of the mixing matrix we immediately see that above speculation, within the defined context, has no consequence because unitarity implies $P_{e e}[\mathrm{Eq} \cdot(\overline{7})]=P_{e e}[\mathrm{Eq} \cdot(13)]$.

IV. In astrophysical environments, such as for neutrinos observed in the supernova 1987a [23,24], it may happen that $m_{3}$ is such that the state $\left|\nu_{3}\right\rangle$ can not escape the astrophysical environment. That is, $p / m$ associated with the $\left|\nu_{3}\right\rangle$ is less than the escape velocity

$$
\text { Non Relativistic: } \quad E<m+\frac{r_{g}}{2 r}
$$


where $r_{g} \equiv 2 G M$ is the gravitational radius of the astrophysical object. Under these circumstances Eq. (13), for a detector at Earth, reduces to:

$$
\begin{aligned}
& P_{\ell \ell^{\prime}}\left(E, L,\left\{\xi_{k}\right\}\right)=\left(U_{\ell^{\prime} 3} U_{\ell 3}\right)^{2} \Theta\left(E-m+\frac{r_{g}}{2 r}\right) \\
& +\left(U_{\ell^{\prime} 1} U_{\ell 1}+U_{\ell^{\prime} 2} U_{\ell 2}\right)^{2}-4 U_{\ell^{\prime} 1} U_{\ell 1} U_{\ell^{\prime} 2} U_{\ell 2} \sin ^{2}\left(\varphi_{21}^{0}\right) .
\end{aligned}
$$

In the above expression $\Theta(\cdots)$ is the Heaviside function, vanishing for its argument less than zero and equal to unity for its argument greater or equal to zero. Note that depending upon how much the argument of $\Theta(\cdots)$ exceeds zero, the arrival of the $\left|\nu_{3}\right\rangle$ could be enormously delayed. Further, if $U_{\ell^{\prime} 3}$ is small, the delayed signal could easily also be too small to be detected. For example, in the Kamiokande detector, an oscillation amplitude $\lesssim 0.5$ would have led, within statistical fluctuations, to as few as one additional event beyond the time windows identified with the 1987A supernova pulse (and so could have have failed to be recognized). This possibility was ignored earlier only due to the cosmological constraints which are now in question.

Now we rewrite Eq. (13) in a form that makes the deviations of result (13) explicit from the corresponding two-flavor scenario with two relativistic mass eigenstates. This new form of Eq. (13) reads:

$$
P_{\ell \ell^{\prime}}\left(E, L,\left\{\xi_{k}\right\}\right)=\Delta_{\ell \ell^{\prime}}-A_{\ell \ell^{\prime}} \sin ^{2}\left(\varphi_{21}^{0}\right)
$$

with $\Delta_{\ell \ell^{\prime}}=\left(U_{\ell^{\prime} 3} U_{\ell 3}\right)^{2}+\left(U_{\ell^{\prime} 1} U_{\ell 1}+U_{\ell^{\prime} 2} U_{\ell 2}\right)^{2}$, or more explicitly

$$
\Delta=\mathbb{1}+\left(\begin{array}{ccc}
\mathrm{s}_{\beta}^{4}+\mathrm{c}_{\beta}^{4}-1 & 2 \mathrm{c}_{\beta}^{2} \mathrm{~s}_{\beta}^{2} \mathrm{~s}_{\psi}^{2} & 2 \mathrm{c}_{\beta}^{2} \mathrm{~s}_{\beta}^{2} \mathrm{c}_{\psi}^{2} \\
2 \mathrm{c}_{\beta}^{2} \mathrm{~s}_{\beta}^{2} \mathrm{~s}_{\psi}^{2} & 2 \mathrm{c}_{\beta}^{2} \mathrm{~s}_{\psi}^{2}\left(\mathrm{c}_{\beta}^{2} \mathrm{~s}_{\psi}^{2}-1\right) & 2 \mathrm{c}_{\beta}^{4} \mathrm{c}_{\psi}^{2} \mathrm{~s}_{\psi}^{2} \\
2 \mathrm{c}_{\beta}^{2} \mathrm{~s}_{\beta}^{2} \mathrm{c}_{\psi}^{2} & 2 \mathrm{c}_{\beta}^{4} \mathrm{c}_{\psi}^{2} \mathrm{~s}_{\psi}^{2} & 2 \mathrm{c}_{\beta}^{2} \mathrm{c}_{\psi}^{2}\left(\mathrm{c}_{\beta}^{2} \mathrm{c}_{\psi}^{2}-1\right)
\end{array}\right)
$$

and

$$
A_{\ell \ell^{\prime}} \equiv 4 U_{\ell^{\prime} 1} U_{\ell 1} U_{\ell^{\prime} 2} U_{\ell 2}
$$

where $\mathbb{1}$ is a $3 \times 3$ identity matrix.

Only when both $\beta$ and $\psi$ vanish does Eq. (18) reduce to the expression for the two flavor scenario with two relativistic mass eigenstates, for then $U(\theta, \beta, \psi)$ becomes block diagonal with no mixing with $\left|\nu_{3}\right\rangle, \Delta(\beta=\psi=0)=\mathbb{1}$, and

$$
A=\left(\begin{array}{ccc}
\mathrm{s}_{2 \theta}^{2} & -\mathrm{s}_{2 \theta}^{2} & 0 \\
-\mathrm{s}_{2 \theta}^{2} & \mathrm{~s}_{2 \theta}^{2} & 0 \\
0 & 0 & 0
\end{array}\right)
$$




\section{FRAMEWORK C: NEUTRINO OSCILLATIONS WITH A SUPERPOSITION OF ONE RELATIVISTIC AND TWO NON-RELATIVISTIC MASS EIGENSTATES}

For completeness, we now consider a physical situation where we have one relativistic, $\left|\nu_{1}\right\rangle$, and two non-relativistic, $\left|\nu_{2}\right\rangle$ and $\left|\nu_{3}\right\rangle$, neutrino mass eigenstates. The configuration of masses is so chosen that $m_{1} \ll m_{2} \leq m_{3}$, and

$$
\frac{\delta m_{32}}{\langle m\rangle} \ll 1 ; \quad \delta m_{32} \equiv m_{3}-m_{2}, \quad\langle m\rangle \equiv\left(m_{3}+m_{2}\right) / 2
$$

Then, arguments similar to those above yield:

$$
\begin{aligned}
P_{\ell \ell^{\prime}}\left(E, L,\left\{\xi_{k}^{\prime}\right\}\right)=\left(U_{\ell^{\prime} 1} U_{\ell 1}\right)^{2}+ & {\left[\left(U_{\ell^{\prime 2}} U_{\ell 2}+U_{\ell^{\prime} 3} U_{\ell 3}\right)^{2}\right.} \\
& \left.-4 U_{\ell^{\prime} 1} U_{\ell 1} U_{\ell^{\prime} 2} U_{\ell 2} \sin ^{2}\left(\zeta_{32}^{0}\right)\right] .
\end{aligned}
$$

In Eq. (23), the first term is now the contribution from the relativistic mass eigenstate, while the second interference term arises from the two non-relativistic mass eigenstates.

The new kinematic phase $\zeta_{32}^{0}$ that appears in Eq. (23) is defined as

$$
\zeta_{32}^{0}=\frac{c^{3}}{2 \hbar} \frac{\delta m_{32}\langle m\rangle L}{\left[2\langle m\rangle c^{2}\left(E-\langle m\rangle c^{2}\right)\right]^{1 / 2}} .
$$

To compare the new kinematic phase that now enters our considerations with Eqs. (8) and (9), we rewrite Eq. (24) as:

$$
\zeta_{32}^{0}=2 \pi \frac{L}{\Lambda_{32}^{\mathrm{osc}}},
$$

with the new flavor-oscillation length given by

$$
\Lambda_{32}^{\mathrm{osc}}==\frac{4 \sqrt{2} \pi \hbar}{\delta m_{32} c}\left(\frac{E-\langle m\rangle c^{2}}{\langle m\rangle c^{2}}\right)^{1 / 2} .
$$

Denoting $\left[2\langle m\rangle\left(E-\langle m\rangle c^{2}\right)\right]^{1 / 2}$ by $\langle p\rangle$, Eqs. (24) and (26) take the simpler form

$$
\zeta_{32}^{0}=\frac{c^{2}}{4 \hbar} \frac{\Delta m_{32}^{2} L}{\langle p\rangle}
$$

and

$$
\Lambda_{32}^{\mathrm{osc}}=\frac{8 \pi \hbar}{c^{2}} \frac{\langle p\rangle}{\Delta m_{32}^{2}}
$$

\footnotetext{
${ }^{1}$ Here, we keep $\hbar$ and $c$ explicit in order that appropriate counterparts of 1.27 may be introduced as dictated by the magnitude of relevant variables.
} 
However, this rewriting should not leave the impression that there is now no $m$-dependence. Unlike the standard ultra-relativistic case, where the $m$-dependence of $E$ disappears in $E \simeq p c$, we now the have an explicit $m$-dependence via $\langle p\rangle=\left[2\langle m\rangle\left(E-\langle m\rangle c^{2}\right)\right]^{1 / 2}$.

The neutrino-flavor oscillation probability now depends on the differences in masses of the non-relativistic mass eigenstates, is independent of the mass of the lowest lying mass eigenstate, and has an explicit dependence on the absolute scale of masses of non-relativistic mass eigenstates via $\langle m\rangle$.

The five neutrino oscillation parameters are: $\xi_{1}^{\prime}=\delta m_{32}, \xi_{2}^{\prime}=\langle m\rangle, \xi_{3}^{\prime}=\theta, \xi_{4}^{\prime}=\beta$, and $\xi_{5}^{\prime}=\psi$.

In astrophysical contexts, Eq. (23) is modified similarly to before (cf., Eq.17)

$$
\begin{aligned}
& P_{\ell \ell^{\prime}}\left(E, L,\left\{\xi_{k}^{\prime}\right\}\right)=\left(U_{\ell^{\prime} 1} U_{\ell 1}\right)^{2}+\Theta\left(E-\langle m\rangle+\frac{r_{g}}{2 r}\right) \\
& \quad \times\left[\left(U_{\ell^{\prime} 2} U_{\ell 2}+U_{\ell^{\prime} 3} U_{\ell 3}\right)^{2}-4 U_{\ell^{\prime} 1} U_{\ell 1} U_{\ell^{\prime} 2} U_{\ell 2} \sin ^{2}\left(\zeta_{32}^{0}\right)\right] .
\end{aligned}
$$

It is again transparent that a superposition of relativistic and non-relativistic mass eigenstates has a a rich structure. Important physical information can be gathered about neutrino properties by carefully analyzing the existing data and designing future neutrino experiments at all feasible energies. If the physically realized scenario occurs in a set of experiments that corresponds to Frameworks $\mathrm{A}+\mathrm{B}$, the mass-parameters that enter the neutrino oscillation phenomenology evolves from dependence on $\Delta m_{21}^{2}$ to dependence on $\Delta m_{21}^{2}$ and $\Delta m_{32}^{2}$. If the physically realized scenario occurs in a set of experiments that corresponds to Frameworks $\mathrm{A}+\mathrm{C}$, the mass-parameters that enter the neutrino oscillation phenomenology change from explicit dependence on $m_{2}$ and $m_{3}$ to $\Delta m_{21}^{2}$ and $\Delta m_{32}^{2}$.

\section{KARMEN ANOMALY: A HINT OF A NON-RELATIVISTIC MASS EIGENSTATE?}

KARMEN continues to see an interesting anomaly in the time distribution of $\nu_{e}$ and $\bar{\nu}_{\mu}$ [25.26]. Tentatively, the KARMEN collaboration interprets this anomaly as an indication of a new neutral particle with its mass and lifetime (electromagnetic decay) as follows: 7

$$
\text { KARMEN Particle X: } m_{x}=33.9 \mathrm{MeV}, \quad \tau_{x}>0.3 \mu \mathrm{s} .
$$

We consider the identification of it with $\left|\nu_{3}\right\rangle$, so that

$$
m_{x}=m_{3}
$$

Within the three flavor neutrino oscillation framework, the expectation value of the mass operator in the state $\left|\nu_{\tau}\right\rangle=\sum_{\jmath} U_{\tau \jmath}\left|\nu_{\jmath}\right\rangle$ is

$$
m\left(\nu_{\tau}\right)=U_{\tau 1}^{2} m_{1}+U_{\tau 2}^{2} m_{2}+U_{\tau 3}^{2} m_{3} .
$$

\footnotetext{
${ }^{2}$ This section evolved in discussions with Dr. Hywel White. We wish to acknowledge his physics contributions, and thank him warmly for sharing his wisdom and thoughts with us.
} 
Guided by the current limits on the remaining neutrino masses given by Eqs. (3) and (4) we assume

$$
U_{\tau 1}^{2} m_{1}+U_{\tau 2}^{2} m_{2} \ll U_{\tau 3}^{2} m_{3}
$$

Using the identification (31) we obtain

$$
\left|U_{\tau 3}\right|<0.82
$$

To exploit the phenomenological consequences of our proposal to identify the KARMEN anomaly with the third mass eigenstate one must now incorporate the life time of $\left|\nu_{3}\right\rangle$ in the above proposed framework. In the context of supernova 1987a such a formalism can be found in the work of Frieman, Haber, and Freese [27].

In reference to the PSI's "search for a neutral particle of mass $33.9 \mathrm{MeV}$ in pion decay" 28

$$
\pi^{+} \rightarrow \mu^{+}+X
$$

if $|X\rangle$ is indeed $\left|\nu_{3}\right\rangle$, the kinematics of the KARMEN anomaly may be significantly different from the kinematics associated with the PSI-considered decay (35). While the details of this difference have not been worked out in detail, it is clear that identifying the KARMEN anomaly with the third mass eigenstate means that the particle $\mathrm{X}$ will be found not only in the decay (35) but also (provided the appropriate energy threshold requirements are met) in the tau, muon, and electron neutrino beams. [ Above the production threshold, the relative $\mathrm{X}$-event rates for these beams are expected to be roughly in the ratio

$$
U_{\tau 3}^{2}: U_{\mu 3}^{2}: U_{e 3}^{2} \quad,
$$

for equal fluxes of $\nu_{\tau}, \nu_{\mu}, \nu_{e}$ respectively.

\section{CONCLUDING REMARKS}

In this paper we have established that interplay of non-relativistic and relativistic effects in neutrino oscillations may play an important role in understanding the existing indications of neutrino oscillations and design of future experiments.

As an alternate hypothesis to the assumption of sterile neutrino we have put forward a theoretical structure that contains in it the (a) the standard three-flavor neutrino oscillation framework for the $\mathrm{GeV}$ data on the zenith angle dependent atmospheric neutrino anomaly, and (b) a three-flavor neutrino oscillation framework with one or two non-relativistic mass eigenstates for part of the $\mathrm{MeV}$ data on neutrino oscillation experiments.

If the particle $\mathrm{X}$, with mass $33.9 \mathrm{MeV}$, of the KARMEN collaboration anomaly is identified with the third neutrino mass eigenstate, then the present limit of $23 \mathrm{MeV}$ upper bound on the tau neutrino mass leads to $\left|U_{\tau 3}\right|<0.82$.

\footnotetext{
3 The PSI experiment may be considered as a search for the KARMEN X in the $\nu_{\mu}$ beam.
} 
Our brief discussion on KARMEN anomaly suggests that one may not only need to relax the assumption on the relativistic nature of all three mass eigenstates, as done in the present paper, but in addition the stability of the neutrino mass eigenstates may no longer be a viable hypothesis. 


\section{REFERENCES}

[1] J. N. Bahcall, Neutrino Astrophysics (Cambridge University Press, Cambridge, 1994).

[2] LSND Collaboration, C. Athanassopoulos et al., Phys. Rev. Lett. 75, 2650 (1995);

LSND Collaboration, C. Athanassopoulos et al., Phys. Rev. C (1996, submitted); nuclex/9605001.

[3] Y. Fukuda et al., Phys. Lett. B 335,237 (1994);

For a comment on the statistical significance of the quoted zenith-angle dependence of the atmospheric neutrino anomaly by Fukuda et al., see D. Saltzberg, Phys. Lett. B 355, 499 (1995);

B. C. Barish, Nucl. Phys. B (Proc. Suppl.) 38, 343 (1995).

[4] G. Börner, The Early Universe: Facts and Fiction (Springer-Verlag, Berlin, 1992).

[5] R. Lieu et al., Science 274, 1335 (1996);

S. Bowyer et al., Science 274, 1338 (1996).

[6] S. Bowyer (private communication, Winter 1996).

[7] G. Schilling, Science 274, 1305 (1996); reporting on "a pair of papers soon to appear in Astronomy and Astrophysics" by H. Arp and H.-D. Radecke.

[8] D. Buskulic et al., Phys. Lett. B 349, 585 (1995).

[9] Particle Data Group, R. M. Barnett et al., Phys. Rev. D 54, 1 (1996).

[10] C. M. Kim and A. Pevsner, Neutrinos in Physics and Astrophysics (Harwood Academic Publishers, 1993).

[11] B. Kayser (with F. Gibrat-Debu and F. Perrier) The Physics of Massive Neutrinos (World Scientific, Singapore, 1989).

[12] R. N. Mohapatra and P. B. Pal, Massive Neutrinos in Physics and Astrophysics (World Scientific, Singapore, 1991).

[13] L. Maiani, Phys. Lett. B 62, 183 (1976).

However, the manner in which rows and columns are labeled and notation for angles is essentially that of Ref. [10, Eq. 6.21].

[14] D. V. Ahluwalia, hep-ph/9612471.

[15] S. P. Mikheyev and A. Yu. Smirnov, Sov. J. Nucl. Phys. 42, 913 (1985);

L. Wolfenstein, Phys. Rev. D 17, 2369 (1978).

[16] D. V. Ahluwalia and C. Burgard, Gen. Rel. and Grav. 28, 1161 (1996). See also, Y. Kojima, gr-qc/9612044;

J. R. Mureika, hep-ph/9612391;

N. Fornengo, C. Giunti, C. W. Kim, and J.Song, hep-ph/9611231;

C. Y. Cardall and G. M. Fuller, hep-ph/9610494;

Y. Grossman and H. Lipkin, gr-qc/9607201;

T. Bhattacharya, S. Habib and E. Mottola, gr-qc/9605074;

B. Jegerlehner, F. Neubig and G. Raffelt, Phys. Rev. D. 54, 1194 (1996). In reference to their "note added" one may wish to consider the LSND implied $\Delta m^{2}$ rather than $\Delta m^{2}$ as implied by the solar neutrino deficit. The effect of the gravitationally induced phases than becomes apparent. ;

D. V. Ahluwalia and C. Burgard, gr-qc/9606031.

[17] D. Píriz, M. Roy, and J. Wudka, Phys. Rev. D 54, 2761 (1996).

[18] See, e.g., J. T. Poltoniemi, hep-ph/9506228. 
[19] G. Zacek et al., Phys. Rev. D 34, 2621 (1986).

[20] G. S. Vidyakin et al., Pis'ma Zh. Eksp. Teor. Fiz. 59, 364 (1994) [English translation: JETP Lett. 59, 390 (1994)].

[21] B. Achkar et al., Nucl. Phys. B 434, 503 (1995).

[22] Z. D. Greenwood et al., Phys. Rev. D 53, 6054 (1996).

[23] K. Hirata et al., Phys. Rev. Lett. 58, 1490 (1987);

R. M. Bionta, Phys. Rev. Lett. 58, 1494 (1987);

M. Aglietta et al., in The Standard Model, The Supernova 1987a, J. Tran Thanh Van, ed., Proceedings of the Leptonic Session of the Twenty-Second Rencontre de Moriond (Editions Frontières, France, 1987);

E. N. Alexeyev et al., in The Standard Model, The Supernova 1987a, J. Tran Thanh Van, ed., Proceedings of the Leptonic Session of the Twenty-Second Rencontre de Moriond (Editions Frontières, France, 1987).

[24] M. Roos, In The Standard Model, The Supernova 1987a, J. Tran Thanh Van ed., Proceedings of the Leptonic Session of the Twenty-Second Rencontre de Moriond (Editions Frontières, France, 1993);

J. N. Bahcall, and S. L. Glashow, Nature 326, 476 (1987).

[25] KARMEN collaboration, B. Armbruster et al. Phys. Lett. B 348, 19 (1995).

[26] G. Drexlin (of KARMEN collaboration) (private communication, Winter 1996).

KARMEN continues to see the anomalous events reported in [25]. While the effect is not a $5 \sigma$ effect yet, its statistical significance has increased since the first published report in Ref. [25].

[27] J. A. Frieman, H. E. Haber, and K. Freese, Phys. Lett. B 200, 115 (1988).

[28] M. Daum et al. Phys. Lett. B 361, 179 (1995). 